\title{
Assessing the risk of waiting for coronary artery bypass graft surgery among patients with stenosis of the left main coronary artery
}

\author{
Jean-François Légaré, Alex MacLean, Karen J. Buth, John A. Sullivan
}

§ See related article page 381

\section{Abstract}

Background: Significant controversy remains over how urgently coronary artery bypass graft surgery (CABG) should be scheduled, particularly for patients with stenosis of the left main coronary artery. Our main objective was to evaluate the safety of waiting for CABG among patients with left main coronary artery disease using a standardized triage system.

Methods: We identified 561 consecutive patients with stenosis of the left main coronary artery who were scheduled to undergo CABG between Apr. 1, 1999, and Mar. 31, 2003. Using standardized triage criteria, patients were assigned to 1 of 4 waiting queues: "emergent," "in-hospital urgent," "out-of-hospital semi-urgent $A^{\prime \prime}$ and "out-of-hospital semi-urgent B." Postoperative outcome measures were in-hospital death from any cause and a composite outcome measure of in-hospital death from any cause, a prolonged requirement for postoperative mechanical ventilation (>24 h) and a prolonged postoperative hospital stay (>9d). Waiting-time variables included the specific queue, whether patients waited longer than the standard time established for each queue and whether patients were upgraded to a more urgent queue. Logistic regression analysis was used to identify independent predictors of the composite outcome; propensity scores (probability of being assigned to a specific queue) were entered into the model to adjust for patient variability among queues.

Results: Of the 561 patients, 65 (11.6\%) were assigned to the emergent group, $343(61.1 \%)$ to the in-hospital urgent group, $91(16.2 \%)$ to the semi-urgent A queue and $62(11.1 \%)$ to the semi-urgent B queue. Four patients $(0.7 \%)$ died while waiting for surgery. The median waiting times were as follows: emergent group, 0 days; in-hospital urgent group, 2 days; 30 days in the semi-urgent $\mathrm{A}$ group and 49 days in the semi-urgent $\mathrm{B}$ group. A total of 52 patients $(9.3 \%)$ were upgraded to a more urgent queue, and 147 patients (26.2\%) waited longer than the standard times for their respective queue. The overall inhospital mortality was $5.5 \%(n=31)$, and the composite outcome was $32.6 \%(n=183)$. Independent predictors of the composite outcome were myocardial infarction within 7 days before surgery, preoperative renal failure, ejection fraction of less than $40 \%$, age greater than 70 years and stenosis of left main coronary artery greater than $70 \%$. Waiting-time variables were associated with neither a significantly higher mortality nor morbidity outcome.

Interpretation: For selected patients with stenosis of the left main coronary artery, waiting for CABG did not appear to be associated with increased mortality or morbidity.

CMAJ 2005;173(4):371-5

$\mathrm{S}$ tenosis of more than $50 \%$ in the left main coronary artery has been a well-accepted indication for coronary artery bypass grafting (CABG). ${ }^{1}$ Surgical intervention in the form of $\mathrm{CABG}$ has been associated with a significant survival benefit, with a median survival exceeding 13 years compared with 6.6 years among patients treated medically. ${ }^{2,3}$

Much of the evidence to date suggests that it is safe to wait for CABG within defined "standard waiting times," with adverse event rates reported to be low. ${ }^{4-8}$ However, these findings are based on relatively small samples, with little consensus on the general terms describing waiting times. In fact, others have found that the risk of cardiac events may increase with the length of waiting time and that patients suffer both physical and emotional stresses. ${ }^{9}$ Unstable angina has also been suggested as an independent predictor of complications while waiting. ${ }^{4,10}$

There remains controversy as to how urgently to schedule CABG procedures. This is especially true for patients with stenosis of the left main coronary artery, as few studies have been done involving this patient cohort. Waiting for CABG has been reported to be safe for the less urgent cases among such patients. ${ }^{4,6}$ There is, however, a lack of clarity as to how to stratify patients safely.,

We conducted this study to evaluate the safety of waiting for $\mathrm{CABG}$ among patients with stenosis of the left main coronary artery assigned to different waiting queues using a standardized triage system. We reviewed the outcomes of all consecutive patients with such stenosis who were triaged for surgery at a single centre in Nova Scotia. The triage system developed and currently used in Nova Scotia was formed on the basis of recommendations from a 1991 provincial task force asked to develop standards for access 
to various cardiovascular services in the province. With the system, patients are assigned to 1 of 4 waiting queues according to their clinical symptoms, stress test results and cardiac anatomy. ${ }^{8,11}$

\section{Methods}

We identified all consecutive patients with stenosis of the left main coronary artery who were scheduled to undergo CABG between Apr. 1, 1999, and Mar. 31, 2003, at a single centre in Halifax. This centre is responsible for all cardiac catheterization procedures and interventions in Nova Scotia, and therefore choosing it for our study allowed us to capture virtually all patients in the province referred for cardiac surgery and followed over time.

Stenosis of the left main coronary artery was defined as narrowing of the artery of $50 \%$ or more, as detected by angiography. Patients admitted for repeat CABG were excluded to avoid having cases of stenosis in patients with a patent graft.

Indications for CABG were reviewed weekly by a group of cardiologists, cardiac surgeons and cardiac radiologists. Individual patients were assigned to a waiting queue on the basis of objective criteria, as previously described. ${ }^{8,11}$ The criteria comprised 2 major determinants (anatomy of coronary artery disease and symptom severity) and 2 minor determinants (left ventricular function and results of noninvasive testing). Patients were assigned to 1 of 4 queues: "emergent," "in-hospital urgent," "out-of-hospital semiurgent A" and "out-of-hospital semi-urgent B." Patients in the emergent queue were those who presented to hospital on an emergency basis and underwent surgery immediately, as clinically indicated. Patients in the in-hospital urgent queue were those who had Canadian Cardiovascular Society (CCS) class IV symptoms and were kept in the hospital before surgery. In the 2 semi-urgent queues, clinically stable patients (CCS class I-III) were discharged home while waiting for surgery and were stratified according to results of objective functional testing. The semi-urgent A group included patients who scored less than 2 metabolic equivalents on a stress test using the standard Bruce protocol, and the semiurgent B group was made up of those who scored between 2 and 5 metabolic equivalents on the exercise stress test.

Patients were assigned the first available surgeon. All patients with worsening symptoms where evaluated by an attending cardiologist, and data in support of reclassification (defined as an upgrade) were reviewed at the next cardiovascular conference. No patients were downgraded.

Nine patients who did not follow the above queuing system (because of patient-driven delay, surgeon-driven delay or referral from another province) were excluded from the analysis.

CABG, performed with or without cardiopulmonary bypass, was performed in a standardized fashion. ${ }^{12}$ The choice of conduits or construction of composite grafts, or both, was based on surgeon preferences rather than on fixed criteria. To achieve a target activated clotting time of more than 450 seconds, heparin was given at a dose of $300 \mathrm{IU} / \mathrm{kg}$ in the cardiopulmonary bypass group and $100 \mathrm{IU} / \mathrm{kg}$ in the beating-heart group. On completion of anastomoses, both groups received protamine sulfate to reverse the effects of the heparin and return the activated clotting time to preoperative levels.

All patients were taken to a dedicated cardiovascular intensive care unit after surgery. Each patient was required to meet standard criteria before extubation and before transfer to the intermediate care unit. Discharged patients were transferred to an intermediate care or general care ward under the care of the same team. All patients were monitored continuously for at least 24 hours after surgery.

All patients received intravenous nitroglycerin infusions (0.1$8 \mu \mathrm{g} / \mathrm{kg}$ per minute) for the first 24 hours unless they were hypotensive (systolic blood pressure $<90 \mathrm{~mm} \mathrm{Hg}$ ). Oral nifedipine therapy (Adalat $10 \mathrm{mg} 4$ times daily, or Adalat XL $30 \mathrm{mg} / \mathrm{d}$ ) was prescribed for patients receiving a radial artery graft; therapy was started on day 1 postoperatively and continued for 3-6 months. Other routine medications included daily ASA and resumption of cholesterol-lowering agents, $\beta$-blockers and angiotensinconverting-enzyme inhibitors, as appropriate.

Waiting-time variables were expressed in days and included the specific waiting queues. Additional waiting-time variables included whether patients waited longer than the standard time established for each queue, and whether patients were upgraded to a more urgent queue. The postoperative outcome measures of interest were in-hospital death from any cause and a composite outcome measure (defined on the basis of previous work ${ }^{8}$ ) composed of in-hospital death from any cause, mechanical ventilation for more than 24 hours postoperatively and a postoperative hospital stay of more than 9 days.

Descriptive statistics included continuous and discrete variables (Appendix 1), which were analyzed accordingly with the use of an unpaired $t$ test, the Wilcoxon rank-sum test, the $\chi^{2}$ test and Fisher's exact test. Statistical significance was defined as a $p$ value of less than 0.05 . A multinomial logistic regression model was used to predict the probability of queue assignment after adjustment for all relevant preoperative patient characteristics. A propensity score (indicating the probability of being in a specific waiting queue) was calculated for each patient. A multinomial logistic regression model was used to identify the independent predictors of the composite outcome, and the propensity score for queue assignment was included in this model to adjust for potential patient variability among queues. The follow-up of all patients undergoing CABG in Halifax, including those involved in our study, was approved by the Capital Health Research Ethics Board.

\section{Results}

A total of 561 consecutive patients with stenosis of the left main coronary artery were identified during the study period. During the same period, 3389 isolated CABG procedures were performed. Patient characteristics are listed in Table 1. A large proportion of the patients were over 70 years old, were male, had CCS class III or IV symptoms and were admitted to hospital with unstable angina. Stenosis of the left main coronary artery greater than $70 \%$ was present in two-thirds of the cases.

Each patient was assigned to a waiting queue (Table 2). Standard waiting times for these queues are as follows: emergent, no delay (0 days); in-hospital urgent, 7 days; outof-hospital semi-urgent A, 21 days; and out-of-hospital semi-urgent $\mathrm{B}, 56$ days. ${ }^{8,11}$ The 343 patients $(61.1 \%)$ in the in-hospital urgent group had a median wait time of 2 days (interquartile range 0-6 d); $46(13.4 \%)$ waited longer than the standard waiting time of 7 days, and $31(9.0 \%)$ had been upgraded from a less urgent queue. Overall, 147 patients $(26.2 \%)$ waited longer than the standard; $52(9.3 \%)$ were 
upgraded to a more urgent queue while they were waiting.

The majority of the procedures were performed with cardiopulmonary bypass; 26 (4.7\%) were off-pump CABG procedures. The mean number of distal anastomoses was 3.2 (standard deviation [SD] 0.9) grafts, and the mean pump time was 109 (SD 42) minutes. A total of 104 patients required an intra-aortic balloon pump preoperatively $(18.5 \%)$; an additional $9(1.6 \%)$ required one intraoperatively (1.6\%); and $5(0.9 \%)$, postoperatively.

\begin{tabular}{lc}
\hline $\begin{array}{l}\text { Table 1: Characteristics of } \mathbf{5 6 1} \text { consecutive patients } \\
\text { with stenosis of the left main coronary artery }\end{array}$ \\
\hline & $\begin{array}{c}\text { No. }(\%) \\
\text { of patients }\end{array}$ \\
Characteristic & $233(41.5)$ \\
\hline Age $>70$ yr & $138(24.6)$ \\
Female & $93(16.6)$ \\
Ejection fraction < 40\% & \\
CCS class & $11(2.0)$ \\
I & $47(8.4)$ \\
II & $173(30.8)$ \\
III & $330(58.8)$ \\
IV & $183(32.6)$ \\
Unstable angina & $72(12.8)$ \\
Myocardial infarction within 7 d & $104(18.5)$ \\
before surgery & \\
Preoperative intra-aortic balloon pump & $111(19.8)$ \\
required & $209(37.3)$ \\
Medical history & $373(66.5)$ \\
Congestive heart failure & $463(82.5)$ \\
Diabetes mellitus & $103(18.4)$ \\
Hypertension & $127(22.6)$ \\
Hypercholesterolemia & $94(16.8)$ \\
Chronic obstructive lung disease & $41(7.3)$ \\
Peripheral vascular disease & \\
Cerebrovascular disease & $375(66.8)$ \\
Preoperative renal failure & \\
Stenosis of left main coronary artery & \\
$50 \%-70 \%$ & \\
$>70 \%$ & \\
\hline Note: CCS class = Canadian Cardiovascular Society angina classification. \\
\end{tabular}

The overall all-cause mortality was $5.5 \%(n=31$, including patients who died while waiting). The mortality was $4.1 \%(6 / 147)$ among patients waiting longer than the standard time for the queue $(p=0.37)$ and $9.6 \%(5 / 52)$ among those who were upgraded $(p=0.17)$. Four patients died while on the waiting list for a total of 833 days, for a mortality of $0.7 \%$ while waiting for surgery. All 4 patients had been waiting at home, with 3 in the semi-urgent A group and 1 in the semi-urgent $B$ group. Two of these patients had been waiting longer than the standard time before they died. The overall mortality appeared to increase with increasing urgency (urgency queue) but did not reach statistical significance (Table 3).

The results of the multinomial logistic regression analysis to identify independent predictors of the composite outcome are shown in Table 4 . A total of 183 patients $(32.6 \%)$ had the composite outcome (Table 3), with 96 (17.1\%) requiring prolonged ventilation and 134 (23.9\%) requiring a prolonged hospital stay. Independent risk factors for the composite outcome were recent myocardial infarction, preoperative renal failure, ejection fraction below $40 \%$, age over 70 years and high-grade stenosis $(>70 \%)$ of the left main coronary artery (Table 4). None of the waiting-time variables were found to be independently associated with the composite outcome. In this fully adjusted model, the c statistic was 0.78 (nonsignificant).

\section{Interpretation}

We evaluated the safety of waiting for CABG among patients with stenosis of the left main coronary artery assigned to waiting queues using a standardized triage system. The overall mortality while waiting for surgery was less than $1 \%$. We found no increase in adverse outcomes among patients assigned to a specific queue, those who waited longer than the standard time or among those who were upgraded to a more urgent queue. A propensity score (indicating the probability of being in a specific waiting queue) was calculated for each patient and was included as a variable in the logistic regression analysis. The rationale for using this approach was to account for as many variables as

Table 2: Waiting-time variables by waiting queue

\begin{tabular}{lcccc}
\hline & \multicolumn{4}{c}{ Waiting queue* } \\
\cline { 2 - 5 } Variable & $\begin{array}{c}\text { Emergent } \\
n=65\end{array}$ & $\begin{array}{c}\text { In-hospital urgent } \\
n=343\end{array}$ & $\begin{array}{c}\text { Out-of-hospital } \\
\text { semi-urgent A } \\
n=91\end{array}$ & $\begin{array}{c}\text { Out-of-hospital } \\
\text { semi-urgent B } \\
n=62\end{array}$ \\
\hline $\begin{array}{l}\text { Standard waiting time, d } \\
\text { Actual waiting time, median (IQR), d }\end{array}$ & 0 & 7 & 21 & 56 \\
$\begin{array}{l}\text { Wait longer than standard waiting } \\
\text { time, no. (\%) of patients }\end{array}$ & $10(15.4)$ & $46(13.4)$ & $67(73.6)$ & $49(27-69)$ \\
$\begin{array}{l}\text { Patient upgraded into more urgent } \\
\text { queue, no. (\%) }\end{array}$ & $8(12.3)$ & $31(9.0)$ & $12(13.2)$ & $24(38.7)$ \\
\hline
\end{tabular}

Note: IQR = interquartile range, $\mathrm{NA}=$ not applicable.

*The number of patients in each queue reflects the number at the end of the study period and includes any patients who were upgraded while waiting for surgery. 
possible that could result in a patient being assigned to a specific queue. Using this approach, we found that the waiting queue did not emerge as an independent predictor of the composite outcome. However, we did find that $26 \%$ of the patients waited longer than the standard time and that $9 \%$ were upgraded to a more urgent queue because of clinical deterioration while waiting for surgery. In fact, $70 \%$ of the patients in the semi-urgent A queue waited longer than the standard 7-day wait. Taken together, our data suggest that our ability to deliver cardiac surgical care in Nova Scotia does not always meet the demands of timely surgery for some patients waiting for CABG.

The triage system used has previously been shown to be equitable for most patients waiting for CABG surgery and to result in few adverse events during the wait. ${ }^{8,11}$ In our study, the overall mortality of less than $1 \%$ among patients waiting for $\mathrm{CABG}$ and the postoperative mortality of $5 \%$ were consistent with previous reports for patients waiting for CABG in Canada, where managed delays are the norm. ${ }^{6,8,11,13}$ Our findings contrast with others that have suggested that stenosis of the left main coronary artery may be associated with an increased risk of death. ${ }^{4,14}$ However, many of these studies were limited by their small size $(n=56-90)$. Supporting our findings of no association with increased mortality were results from a large study involving more than 5864 consecutive patients waiting for CABG. ${ }^{10}$ Our reported independent predictors of the composite outcome were also consistent with published predictors of adverse outcomes following surgery and included recent myocardial infarction, preoperative renal insufficiency, low ejection fraction and advanced age..$^{15,16}$

Our study included patients who were assigned to a waiting queue for $C A B G$ after cardiac catheterization. It was therefore not designed to evaluate the safety of waiting for timely cardiology referral or for cardiac catheterization, all of which occur before surgical referral. ${ }^{17,18} \mathrm{We}$ also attempted to address the low event rate by using a composite outcome that included in-hospital death from any cause plus 2 important surrogates for morbidity: prolonged mechanical ventilation and prolonged hospital stay. ${ }^{8}$ However, despite our inability to demonstrate that waiting was independently predictive of increased mortality, it is difficult to ignore the fact that 4 patients died while waiting at home for surgery. Thus, our study may have been significantly underpowered to demonstrate mortality differences.

It remains unclear what the acceptable rate should be for adverse events in managed waiting strategies. In the current era of limited resources, the reporting of data such as ours is an important mechanism for evaluating resource allocation and giving feedback to government. Similarly, it is now possible to prospectively follow changes in resource allocation and their impact on health care delivery in order to minimize delays.

Table 4: Results of multinomial logistic regression analysis to identify independent predictors of composite outcome*

\begin{tabular}{|c|c|}
\hline Variable & Odds ratio $(95 \% \mathrm{Cl})$ \\
\hline $\begin{array}{l}\text { Myocardial infarction within } 7 \mathrm{~d} \\
\text { before surgery }\end{array}$ & $3.7(2.0-6.9) \dagger$ \\
\hline Preoperative renal failure & $2.9(1.3-6.3) \dagger$ \\
\hline Ejection fraction $<40 \%$ & $2.3(1.3-3.8) \dagger$ \\
\hline Age $>70 \mathrm{yr}$ & $2.1(1.4-3.2) \dagger$ \\
\hline Stenosis of left main coronary artery $>70 \%$ & $1.7(1.05-2.7) \dagger$ \\
\hline Wait longer than standard waiting time & $0.7(0.4-1.2)$ \\
\hline Propensity score (balancing score)‡ & $0.9(0.6-1.5)$ \\
\hline \multicolumn{2}{|l|}{ Queue assignment } \\
\hline Emergent & $2.5(0.95-6.5)$ \\
\hline In-house urgent & $0.9(0.4-1.9)$ \\
\hline Out-of-hospital semi-urgent A & $0.7(0.3-1.6)$ \\
\hline
\end{tabular}

\begin{tabular}{|c|c|c|c|c|c|}
\hline \multirow[b]{2}{*}{ Outcome } & \multicolumn{5}{|c|}{ Waiting queue* } \\
\hline & $\begin{array}{c}\text { Emergent } \\
n=65\end{array}$ & $\begin{array}{c}\text { In-hospital } \\
\text { urgent } \\
n=343\end{array}$ & $\begin{array}{c}\text { Out-of-hospital } \\
\text { semi-urgent A } \\
n=91\end{array}$ & $\begin{array}{c}\text { Out-of-hospital } \\
\text { semi-urgent B } \\
n=62\end{array}$ & $\begin{array}{c}\text { All patients } \\
n=561\end{array}$ \\
\hline \multicolumn{6}{|l|}{ Death while waiting } \\
\hline No. (\%) of patients & 0 & 0 & $3 \quad(3.3)$ & 1 (1.6) & $4 \quad(0.7)$ \\
\hline $95 \% \mathrm{Cl}$ & $0-5.5$ & $0-1.1$ & $0.7-9.3$ & $0.04-8.7$ & $0.2-1.8$ \\
\hline \multicolumn{6}{|c|}{ In-hospital death, all causes } \\
\hline No. $(\%)$ of patients & $6 \quad(9.2)$ & $18 \quad(5.2)$ & $5 \quad(5.5)$ & $2(3.2)$ & $31 \quad(5.5)$ \\
\hline $95 \% \mathrm{Cl}$ & $2.2-16.3$ & $2.9-7.6$ & $1.8-12.4$ & $0.4-11.2$ & $3.6-7.4$ \\
\hline \multicolumn{6}{|l|}{ Composite outcome $\dagger$} \\
\hline No. (\%) of patients & $41(63.1)$ & $111(32.4)$ & $16(17.6)$ & $15(24.2)$ & $183(32.6)$ \\
\hline $95 \% \mathrm{Cl}$ & $51.3-74.8$ & $27.4-37.3$ & $9.8-25.4$ & $13.5-34.9$ & $28.7-36.5$ \\
\hline
\end{tabular}

Note: $\mathrm{Cl}=$ confidence interval.

*The number of patients in each queue reflects the number at the end of the study period and includes any patients who were upgraded while waiting for surgery. tComprised in-hospital death from any cause, prolonged mechanical ventilation ( $>24$ hours) postoperatively and prolonged hospital stay ( $>9$ days) postoperatively. 
This article has been peer reviewed.

From the Division of Cardiac Surgery, Department of Surgery, Dalhousie University, Halifax, NS

\section{Competing interests: None declared.}

Contributors: Jean-François Légaré drafted the article and contributed substantially to the study conception and design and to the analysis and interpretation of the data. Alex MacLean contributed to the acquisition, validation and analysis of the data. Karen Buth contributed substantially to the analysis of the data. John Sullivan contributed substantially to the study design. All of the authors critically revised the article and approved the final version for publication.

\section{References}

1. Naylor CD, McGlynn EA, Leape LL, Pinfold SP, Bernstein SJ, Hilborne LH, et al. Coronary angiography and revascularization: defining procedural indications through formal group processes. The Canadian Revascularization Panel, the Canadian Coronary Angiography Panel. Can 7 Cardiol 1994;10:41-8.

2. Caracciolo EA, Davis KB, Sopko G, Kaiser GC, Corley SD, Schaff H, et al. Comparison of surgical and medical group survival in patients with left main coronary artery disease. Long-term CASS experience. Circulation 1995;91: 2325-34.

3. Eagle KA, Guyton RA, Davidoff R, Ewy GA, Fonger J, Gardner TJ, et al. ACC/AHA guidelines for coronary artery bypass graft surgery: executive summary and recommendations. A report of the American College of Cardiology/American Heart Association Task Force on Practice Guidelines (Committee to Revise the 1991 Guidelines for Coronary Artery Bypass Graft Surgery). Circulation 1999;100:1464-80.

4. Da Rocha AS, da Silva PR. Can patients with left main coronary artery disease wait for myocardial revascularization surgery? Arq Bras Cardiol 2003;80: 191-3.

5. Koomen EM, Hutten BA, Kelder JC, Redekop WK, Tijssen JG, Kingma JH. Morbidity and mortality in patients waiting for coronary artery bypass surgery. Eur 7 Cardiothorac Surg 2001;19:260-5.

6. Maziak DE, Rao V, Christakis GT, Buth KJ, Sever J, Fremes SE, et al. Can patients with left main stenosis wait for coronary artery bypass grafting? Ann Thorac Surg 1996;61:552-7.

7. Naylor CD, Baigrie RS, Goldman BS, Basinski A. Assessment of priority for coronary revascularisation procedures. Revascularisation Panel and Consensus Methods Group. Lancet 1990;335:1070-3.

8. Ray AA, Buth KJ, Sullivan JA, Johnstone DE, Hirsch GM. Waiting for cardiac surgery: results of a risk-stratified queuing process. Circulation 2001;104: I92-8.

9. Sampalis J, Boukas S, Liberman M, Reid T, Dupuis G. Impact of waiting time on the quality of life of patients awaiting coronary artery bypass grafting. CMA7 2001;165(4):429-33.

10. Rexius H, Brandrup-Wognsen G, Oden A, Jeppsson A. Mortality on the waiting list for coronary artery bypass grafting: incidence and risk factors. Ann Thorac Surg 2004;77:769-74.

11. Cox JL, Petrie JF, Pollak PT, Johnstone DE. Managed delay for coronary artery bypass graft surgery: the experience at one Canadian center. 7 Am Coll Cardiol 1996;27:1365-73.

12. Legare JF, Buth KJ, King S, Wood J, Sullivan JA, Friesen CH, et al. Coronary bypass surgery performed off pump does not result in lower in-hospital morbidity than coronary artery bypass grafting performed on pump. Circulation 2004;109:887-92.

13. Naylor CD, Morgan CD, Levinton CM, Wheeler S, Hunter L, Klymciw K, et al. Waiting for coronary revascularization in Toronto: 2 years' experience with a regional referral office. CMA7 1993;149(7):955-62.

14. Naylor CD, Szalai JP, Katic M. Benchmarking the vital risk of waiting for coronary artery bypass surgery in Ontario. CMA7 2000;162(6):775-9.

15. Bardell T, Legare JF, Buth KJ, Hirsch GM, Ali IS. ICU readmission after cardiac surgery. Eur 7 Cardiothorac Surg 2003;23:354-9.

16. Légaré JF, Hirsch GM, Buth KJ, MacDougall C, Sullivan JA. Preoperative prediction of prolonged mechanical ventilation following coronary artery bypass grafting. Eur 7 Cardiothorac Surg 2001;20:930-6.

17. Kaul P, Armstrong PW, Chang WC, Naylor CD, Granger CB, Lee KL, et al. Long-term mortality of patients with acute myocardial infarction in the United States and Canada: comparison of patients enrolled in Global Utilization of Streptokinase and t-PA for Occluded Coronary Arteries (GUSTO)-I. Circulation 2004;110:1754-60.

18. Pilote L, Joseph L, Belisle P, Penrod J. Universal health insurance coverage does not eliminate inequities in access to cardiac procedures after acute myocardial infarction. Am Heart 7 2003;146:1030-7.

Correspondence to: Dr. Jean-François Légaré, Division

of Cardiac Surgery, New Halifax Infirmary, Rm. 2269,

1796 Summer St., Halifax NS B3H 3A7; fax 902 473-4448;

jean.legare@cdha.nshealth.ca

\section{Appendix 1: Definitions of key variables used in the analysis of patients waiting for coronary artery bypass grafting}

Variable Definition

Unstable angina Intravenous nitroglycerine required until the time of surgery, or intra-aortic balloon pump required preoperatively because of angina, or both

Preoperative renal Serum creatinine level $>177 \mathrm{mmol} / \mathrm{L}$

failure

Cerebrovascular History of transient ischemic attack, stroke,

disease carotid artery surgery or documented stenosis of the carotid artery

Peripheral vascular History of aneurysm or occlusive vascular disease, disease or both, with or without prior peripheral vascular surgery 